\title{
Fundamental Efficiency Bounds for the Conversion of a Radiative Heat Engine's Own Emission into Work
}

\author{
Andreas Pusch $\odot,{ }^{1, *}$ Jeffrey M. Gordon, ${ }^{2,3}$ Alex Mellor, ${ }^{4, \dagger}$ Jacob J. Krich, ${ }^{5}$ and \\ Nicholas J. Ekins-Daukes ${ }^{1}$ \\ ${ }^{1}$ School of Photovoltaic and Renewable Engineering, University of New South Wales, Sydney, \\ New South Wales 2052, Australia \\ ${ }^{2}$ Department of Solar Energy and Environmental Physics, Jacob Blaustein Institutes for Desert Research, \\ Ben-Gurion University of the Negev, Sede Boqer Campus, 8499000, Israel \\ ${ }^{3}$ School of Mechanical and Chemical Engineering, University of Western Australia, Perth, \\ Western Australia 6009, Australia \\ ${ }^{4}$ Department of Physics, Imperial College London, London SW7 2AZ, United Kingdom \\ ${ }^{5}$ Department of Physics, University of Ottawa, Ottawa, Ontario KIN 6N5, Canada
}

(Received 14 June 2019; revised manuscript received 15 November 2019; published 6 December 2019; corrected 19 October 2020)

\begin{abstract}
The thermoradiative diode is a novel heat engine that converts athermal radiative emission from a hot converter to a colder environment into work. This stands in contrast to essentially all radiative heat engines realized to date, where an external hot reservoir radiates toward a converter that resides at (and can be reversibly coupled to) a cold reservoir. We derive the fundamental bounds on conversion efficiency and power production for generalized far-field hot-side heat engines with radiative exchange that can be black body (thermal) or, more generally, athermal. This is followed by the corresponding derivation for the rich landscape of performance bounds for the specific case of thermoradiative diodes, including why the Landsberg-efficiency limit associated with heat engines that include radiative exchange can be surpassed, and how the ultimate Carnot-efficiency limit could be approached.
\end{abstract}

DOI: 10.1103/PhysRevApplied.12.064018

\section{INTRODUCTION}

Establishing fundamental upper limits on the efficiency with which engines can convert heat to work quantifies the potential room for improvement for any converter technology. The ultimate limit for an engine operating between a hot reservoir of temperature $T_{\text {hot }}$ and a cold reservoir of temperature $T_{\text {cold }}$ is the Carnot efficiency $\eta_{\text {Carnot }}=1-T_{\text {cold }} / T_{\text {hot }}$, predicated on reversible processes and reservoirs of infinite capacity. Converters that include far-field radiative exchange are of special interest due to solar applications. Their constrained upper bound is the eponymous Landsberg efficiency $\eta_{\text {Landsberg }}[1]$ [see Eq. (5) derived later].

In essentially all radiative heat engines realized to date, the converter resides at (and can be reversibly coupled

\footnotetext{
*a.pusch@unsw.edu.au

${ }^{\dagger}$ Now at Naked Energy Ltd., Basepoint Business Centre, Metcalf Way, Crawley RH11 7XX, United Kingdom.

Published by the American Physical Society under the terms of the Creative Commons Attribution 4.0 International license. Further distribution of this work must maintain attribution to the author(s) and the published article's title, journal citation, and DOI.
}

to) the cold reservoir and accepts thermal radiation from a hot reservoir [Fig. 1(a)]. It converts absorbed thermal radiation into work. The Landsberg limit relies on these assumptions(whereas the Carnot limit fundamentally can never be exceeded). The thermoradiative diode (TRD) is a distinct complement to the solar cell in that the TRD resides at the hot reservoir and generates electrical power from its radiative emission to a colder environment [Fig. 1(b)] [2,3]. Although solar applications provided the original motivation for heat engines with radiative exchange, the TRD - and the class of novel hot-side radiative heat engines in which it is subsumed - is a converter of a different nature with dissimilar applications, including power generation from a device at ambient temperature that rejects heat radiatively to the sky [4] and industrialwaste heat recovery through an engine radiating waste heat into a colder environment. Comparisons of the performance of current commercial silicon solar cells against the idealized limit of a TRD with a hot reservoir at a temperature of at least $500 \mathrm{~K}$ show comparable efficiencies and power densities in both cases [3], with results for the TRD depending strongly on the temperature of the hot reservoir and material quality.

While an operating photovoltaic absorbs more photons than it emits, a TRD operates by negative luminescence: 
(a) cold-side converter

(b) hot-side converter
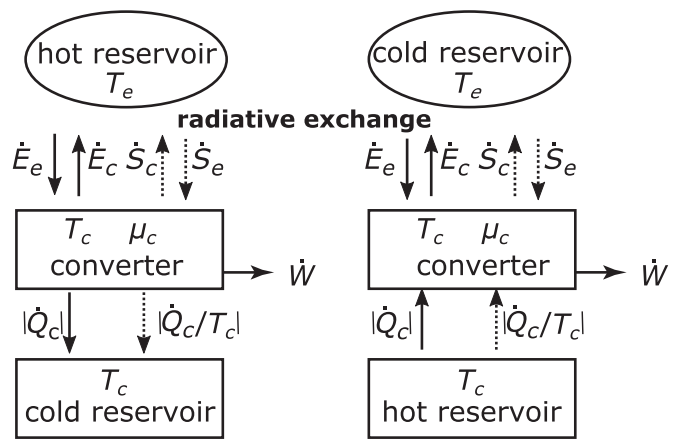

FIG. 1. Heat engines with radiative exchange of energy (solid arrows) and entropy (dashed arrows), where a converter at temperature $T_{c}$ and chemical potential $\mu_{c}$ resides at (and is reversibly coupled to) (a) a cold reservoir (basically all conventional radiative heat engines) and (b) a hot reservoir (as for the TRD), where direct interaction between the two heat reservoirs is excluded. $T_{c}$ refers to the converter temperature in all cases, and $T_{e}$ refers to the environment, which is at a different temperature; that is, a hot reservoir in (a) and a cold reservoir in (b).

the number of emitted photons exceeds the number of absorbed photons, drawing current from an external source and pushing the diode into reverse bias $[3,5,6]$. Both current and voltage have the opposite sign (opposite direction) relative to a (cold-side) photovoltaic cell (see Fig. 2). Power (defined here as negative for power generation and positive for power consumption) is the product of voltage $V$ and current $I$. For a solar cell, $V>0$ and $I<0$, whereas for the TRD, $V<0$ and $I>0$. In a solar cell, excess electron and hole populations are created by radiative absorption, which allows a current to flow against a positive bias.

After reviewing the thermodynamic basis for the Landsberg efficiency, we derive basic upper bounds on the performance of heat engines residing at the hot reservoir and then focus on how these bounds pertain to TRDs, where radiative emission is athermal (i.e., with nonzero chemical

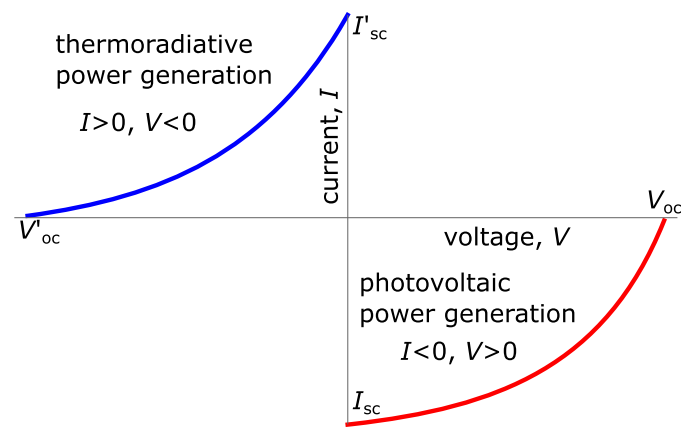

FIG. 2. Dependence of current $I$ on voltage $V$ for a solar cell and a TRD. Subscripts "sc" and "oc" denote "short circuit" and "open circuit," respectively. potential, $\mu$, in contrast to black-body radiation, for which $\mu=0$ ). A somewhat-surprising finding is that this radiative heat engine can, in principle, approach the Carnot limit (and thereby exceed the Landsberg limit). This theoretical possibility is moderated by the extreme sensitivity of device performance to luminescent efficiency and to device imperfections.

\section{LANDSBERG EFFICIENCY}

We review the Landsberg derivation $[7,8]$. For a black body of temperature $T$, the energy flux density is $\sigma T^{4}$ and the entropy flux density is $4 / 3 \sigma T^{3}$. We define the heat flux density $\dot{Q}_{c}$ to be the heat flowing into the converter, as given by the arrow in Fig. 1(b). With this convention, $\dot{Q}_{c}$ is negative for the cold-side converter and positive for the hot-side converter, and we can write the energy and entropy balances for both systems as

$$
\begin{gathered}
\dot{Q}_{c}=\dot{E}_{c}-\dot{E}_{e}+\dot{W}, \\
\dot{S}_{\text {gen }}=-\frac{\dot{Q}_{c}}{T_{c}}+\dot{S}_{c}-\dot{S}_{e},
\end{gathered}
$$

where $S_{\text {gen }}$ is the entropy generation inside the converter. From this we get the maximum extractable work $\dot{W}_{\max }$ or, equivalently, the Helmholtz free energy flux density $\dot{F}$ by setting the entropy generated in the converter to $\dot{S}_{\text {gen }}=0$ :

$$
\begin{aligned}
\dot{F} & =\dot{W}_{\max }=\left(\dot{E}_{e}-T_{c} \dot{S}_{e}\right)-\left(\dot{E}_{c}-T_{c} \dot{S}_{c}\right) \\
& =\sigma\left[\left(T_{e}^{4}-T_{c} \frac{4}{3} T_{e}^{3}\right)-\left(T_{c}^{4}-\frac{4}{3} T_{c}^{4}\right)\right] \\
& =\sigma\left(T_{e}^{4}+\frac{1}{3} T_{c}^{4}-\frac{4}{3} T_{c} T_{e}^{3}\right),
\end{aligned}
$$

where $\sigma$ denotes the Stefan-Boltzmann constant with the symbols defined as in Fig. 1. For a solar-energy converter, conversion efficiency is defined as $\dot{W}_{\max }$ relative to the radiative input $\sigma T_{e}^{4}$, yielding

$$
\eta_{\text {Landsberg }}=\frac{\dot{W}_{\max }}{\sigma T_{e}^{4}}=1-\frac{4}{3} \frac{T_{c}}{T_{e}}+\frac{1}{3} \frac{T_{c}^{4}}{T_{e}^{4}}<\eta_{\text {Carnot }} .
$$

The Landsberg limit is thus the limit for a solar-energy converter that generates no additional entropy beyond that intrinsic to radiative exchange.

Now consider the complementary case where the converter is at the hot side (not germane for solar conversion), using radiative emission to a cold environment to produce work. The maximum conversion efficiency is now defined in relation to the heat flux from the hot reservoir to the converter and is given by $\eta_{\text {hot }}=\dot{W}_{\max } / \dot{Q}_{c}$. Combining Eqs. (4) and (1) for a black body leads to a hot-side analog of the 
Landsberg efficiency for endoreversible converters,

$$
\begin{aligned}
\eta_{\mathrm{hot}} & =\frac{\dot{W}_{\max }}{Q_{c}}=\frac{T_{e}^{4}+(1 / 3) T_{c}^{4}-(4 / 3) T_{c} T_{e}^{3}}{(4 / 3)\left(T_{c}^{4}-T_{c} T_{e}^{3}\right)} \\
& =\frac{1}{4} \eta_{\text {Carnot }} \frac{1+2\left(T_{e} / T_{c}\right)+3\left(T_{e} / T_{c}\right)^{2}}{1+T_{e} / T_{c}+\left(T_{e} / T_{c}\right)^{2}}
\end{aligned}
$$

such that $\eta_{\text {hot }}$ cannot exceed 0.25 [9], the limiting value for $T_{e} / T_{c} \rightarrow 0$, where $\eta_{\text {Carnot }} \rightarrow 1$. In the limit of small temperature differences, the efficiency can approach half the Carnot efficiency. $T_{e}<T_{c}$ with the converter at the hot reservoir, so $\eta_{\text {Carnot }}=1-T_{e} / T_{c}$.

\section{EFFICIENCY BOUNDS FOR ATHERMAL RADIATION}

The limit of Eq. (6) is valid for converters that emit black-body radiation, but a higher efficiency is possible for hot-side converters if we consider non-black-bodies. To generalize this bound to athermal radiative emission, we start with the generalized form of Planck's law [10] for an emitter with an abrupt band gap of energy $E_{g}$ (i.e., absorptivity $a=1$ for $E_{\mathrm{ph}}>E_{g}$ and $a=0$ for $E_{\mathrm{ph}}<E_{g}$, with photon energy $\left.E_{\mathrm{ph}}\right)$. The spectrum-integrated energy flux density $\dot{E}$, particle flux density $\dot{N}$, and grand potential flux density $\dot{\Omega}(\dot{\Omega}=\dot{E}-T \dot{S}-\mu \dot{N})$ are given by [11]

$$
\begin{aligned}
& \dot{E}\left(\mu, E_{g}, T\right)=\frac{2 \pi}{c^{2} h^{3}} \int_{E_{g}}^{\infty} \frac{E_{\mathrm{ph}}^{3}}{e^{\left(E_{\mathrm{ph}}-\mu\right) / k T}-1} d E_{\mathrm{ph}}, \\
& \dot{N}\left(\mu, E_{g}, T\right)=\frac{2 \pi}{c^{2} h^{3}} \int_{E_{g}}^{\infty} \frac{E_{\mathrm{ph}}^{2}}{e^{\left(E_{\mathrm{ph}}-\mu\right) / k T}-1} d E_{\mathrm{ph}} \\
& \dot{\Omega}\left(\mu, E_{g}, T\right)=\frac{2 \pi k T}{c^{2} h^{3}} \int_{E_{g}}^{\infty} E_{\mathrm{ph}}^{2} \ln \left(1-e^{\left(\mu-E_{\mathrm{ph}}\right) / k T}\right) d E_{\mathrm{ph}},
\end{aligned}
$$

where $k$ is Boltzmann's constant, $c$ is the speed of light in a vacuum, and $h$ is Planck's constant. For electrical converters, voltage (or bias) $V=\mu / q$ (assuming infinite carrier mobility [10]), where $q$ is the electron charge.

In any radiative hot-side converter (of which the TRD is one example), the extractable work is a consequence of the free energy generated by the emission process [see Eq. (3)]. So, increasing the ratio of $\dot{W}_{\max }$ to $\dot{Q}_{c}$ requires increasing the ratio of emitted-entropy flux density $\dot{S}_{c}$ to emitted-energy flux density $\dot{E}_{c}$ [5]. We can motivate this requirement by considering the limit in which $T_{e} / T_{c} \rightarrow 0$, where we can ignore $E_{e}$ and $S_{e}$ in $\eta_{\text {hot }}$. In that limit, $\eta_{\text {hot,max }} \rightarrow 1-E_{c} /\left(T_{c} S_{c}\right)$, and we see that increasing $S_{c} / E_{c}$ increases the efficiency. A similar benefit occurs away from the $T_{e} / T_{c} \rightarrow 0$ limit. For black-body emission, the ratio between emitted-entropy flux and emitted-energy flux is fixed at $4 /\left(3 T_{c}\right)$, which sets the stringent limit of Eq. (6).
In contrast, the entropy flux density of radiative emission from the TRD (i.e., athermal radiation) depends on both $E_{g}$ and $\mu$ :

$$
\dot{S}_{c}=\frac{\dot{E}\left(\mu, E_{g}, T_{c}\right)-\mu \dot{N}\left(\mu, E_{g}, T_{c}\right)-\dot{\Omega}\left(\mu, E_{g}, T_{c}\right)}{T_{c}} .
$$

For an engine residing at the cold reservoir (e.g., a solar cell), the internal bias is positive. Hence, the second term in the numerator in Eq. (10) is negative and cannot increase the entropy-to-energy ratio, which determines fundamental efficiency limits. But for an engine residing at the hot reservoir, a reverse bias can be generated that turns the second term in the numerator in Eq. (10) into a positive contribution to the emitted entropy and increases the entropy-to-energy ratio of radiative emission and hence the available free energy in the engine. Put differently, the negative bias decreases the radiative flux without influencing the spectral width, which is determined by the temperature. This is opposite to the operation of a light-emitting diode at positive bias, which increases the radiative flux at constant spectral width and thereby decreases the entropy-to-energy ratio.

We can now generalize the black-body analog to the Landsberg efficiency for hot-side radiative engines [Eq. (6)] to an emitter under reverse bias. Using Eqs. (7)-(9) and the first and second laws of thermodynamics, we obtain

$$
\eta_{\text {hot,max }}=\frac{-\mu \dot{N}_{c}-\dot{\Omega}_{c}+\dot{E}_{e}-\left(T_{c} / T_{e}\right)\left(\dot{E}_{e}-\dot{\Omega}_{e}\right)}{-\mu \dot{N}_{c}-\dot{\Omega}_{c}+\dot{E}_{c}-\left(T_{c} / T_{e}\right)\left(\dot{E}_{e}-\dot{\Omega}_{e}\right)} .
$$

$\eta_{\text {hot,max }}$ is plotted in Fig. 3 as a function of bias at $T_{e} / T_{c}=$ 0.1 for several values of $E_{g}$. $\eta_{\text {hot,max }}$ increases with negative bias because $\dot{S}_{c}$ decreases more slowly with bias than $\dot{E}_{c}$ [a consequence of the additional $-\mu \dot{N}_{c}$ term in Eq. (11)].

Equation (11) is a fundamental limit for a far-field radiative hot-side heat engine (the solid curves in Fig. 3) rather than a limit for the specific TRD heat engine, which is shown by the dashed curve and is derived in the next section. For materials that support surface polaritons - and when fabrication methods can accurately produce ultrasmall gaps between surfaces (smaller than the thermal wavelength) - near-field radiative transfer can create the possibility of higher rates of energy transfer and different rates of entropy transfer than black-body exchange, which in turn can result in larger maximum power output and conversion efficiency $[12,13]$. We do not consider that case here.

\section{THERMORADIATIVE DIODE}

The power from the TRD is obtained from its currentvoltage relation (following from the standard detailedbalance analysis in the radiative limit in which each 


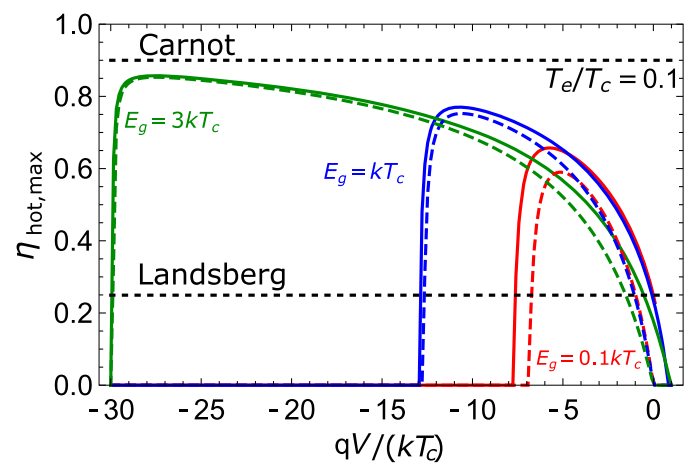

FIG. 3. The fundamental efficiency limit for a radiative hotside heat engine (solid curves) and the corresponding bound for an ideal TRD (dashed curves) as a function of bias normalized to $k T_{c} / q$ for three values of $E_{g}$ at $T_{e} / T_{c}=0.1$. $\eta_{\text {Carnot }}$ is indicated by the upper horizontal dotted black line and the hot-side Landsberg efficiency of Eq. (6) $\eta_{\text {hot }}=24.9 \%$ is indicated by the lower horizontal dotted black line.

electron-hole pair that is lost corresponds to the emission of one photon [14])

$$
I(V)=\dot{N}\left(q V, E_{g}, T_{c}\right)-\dot{N}\left(0, E_{g}, T_{e}\right)
$$

as the product of $I$ and $V$ with use of Eq. (8). The resulting efficiencies, shown in Fig. 3, are lower than the fundamental limit. Equation (12) describes an ideal TRD, meaning a perfect external luminescent efficiency and infinite carrier mobility. Sample $I-V$ curves are presented later as part of the evaluation of the impact of device nonidealities.

Physical transparency and insight can be gained from the closed-form solutions that follow from invoking the Boltzmann approximation $\left[e^{\left(E_{\mathrm{ph}}-\mu\right) / k T} \gg 1\right.$ in Eqs. (7)-(9)], which is accurate for large band gaps and/or large negative bias and introduces only small inaccuracies even for band gaps as small as $E_{g} \approx k T_{c}$. Equations (7)-(9) for the fluxes then become

$$
\begin{aligned}
\dot{E}\left(\mu, E_{g}, T\right)= & \frac{2 \pi(k T)^{4}}{c^{2} h^{3}}\left[\left(\frac{E_{g}}{k T}\right)^{3}+3\left(\frac{E_{g}}{k T}\right)^{2}\right. \\
& \left.+6 \frac{E_{g}}{k T}+6\right] e^{\left(\mu-E_{g}\right) / k T}, \\
\dot{N}\left(\mu, E_{g}, T\right)= & \frac{2 \pi(k T)^{3}}{c^{2} h^{3}}\left[\left(\frac{E_{g}}{k T}\right)^{2}+2 \frac{E_{g}}{k T}+2\right] e^{\left(\mu-E_{g}\right) / k T}, \\
\dot{\Omega}\left(\mu, E_{g}, T\right)= & -k T \dot{N}\left(\mu, E_{g}, T\right) .
\end{aligned}
$$

With this approximation the voltage at maximum power, $V_{\mathrm{mpp}}$, of the TRD is the solution to

$$
\left(1+\frac{q V_{\mathrm{mpp}}}{k T_{c}}\right) e^{q V_{\mathrm{mpp}} / k T_{c}} \dot{N}\left(0, E_{g}, T_{c}\right)=\dot{N}\left(0, E_{g}, T_{e}\right),
$$

which is

$$
V_{\mathrm{mpp}}=\frac{k T_{c}}{q}\left[\mathrm{~W}\left(\frac{e \times \dot{N}\left(0, E_{g}, T_{e}\right)}{\dot{N}\left(0, E_{g}, T_{c}\right)}\right)-1\right]
$$

with the Lambert $\mathrm{W}$ function defined as the solution to $z=\mathrm{W}\left(z e^{z}\right)$. This leads to $V_{\mathrm{mpp}} \approx-k T_{c}$ when $\dot{N}\left(q V=0, E_{g}, T_{e}\right) \ll N\left(q V=0, E_{g}, T_{c}\right)$.

In the limit of large $E_{g}$, Eqs. (13)-(15) can be further simplified to obtain an open-circuit voltage $V_{\mathrm{oc}}=E_{g}\left(1-T_{c} / T_{e}\right)$. An approximation to the voltage at the maximum efficiency is given by

$$
q V_{\max } \approx E_{g}\left(1-T_{c} / T_{e}\right)+T_{c} \ln \frac{T_{c}}{T_{e}}
$$

resulting in an efficiency

$\eta_{\max } \approx \frac{1+T_{e} / T_{c}-T_{e} / E_{g} \ln T_{c} / T_{e}}{1-T_{e} / E_{g} \ln T_{c} / T_{e}} \approx 1-T_{e} / T_{c}=\eta_{\text {Carnot }}$

Thus, as the emitted radiation moves toward becoming pure entropy, the Carnot limit can be approached. This is an intriguing contraposition to the laser limit in cold-side radiative engines, where the coherent radiative emission carries no entropy, becomes pure work, and hence can theoretically be converted to any other form of work with unity efficiency [15].

The efficiency-power trade-off noted above is akin to that observed for essentially all thermodynamic engines [16] but not to that observed for photovoltaics, for which efficiency is defined as the maximum power generated relative to the solar radiation (hot reservoir) impinging on the converter, since the solar radiation itself is a free resource. The TRD's power-efficiency characteristic is graphed in Fig. 4 for several values of $E_{g}$ at $T_{e} / T_{c}=0.1$.

There are subtle opposing trends in the dependence on $E_{g} / T_{c}$ of the efficiency at maximum power in contrast to the maximum attainable efficiency, which can be explained by the limitation on the maximum achievable negative bias, which becomes smaller with increasing radiation absorption from the environment.

\section{NONIDEALITIES}

To determine the efficiency and power in practical TRDs, one needs to evaluate the influence of nonidealities, most prominently nonradiative processes, which, for the TRD, constitute the nonradiative generation of charge carriers. TRD power generation depends on net photon emission to the cold environment even as a reverse bias decreases carrier recombination. But if charge carriers are generated nonradiatively, then the negative bias necessary 

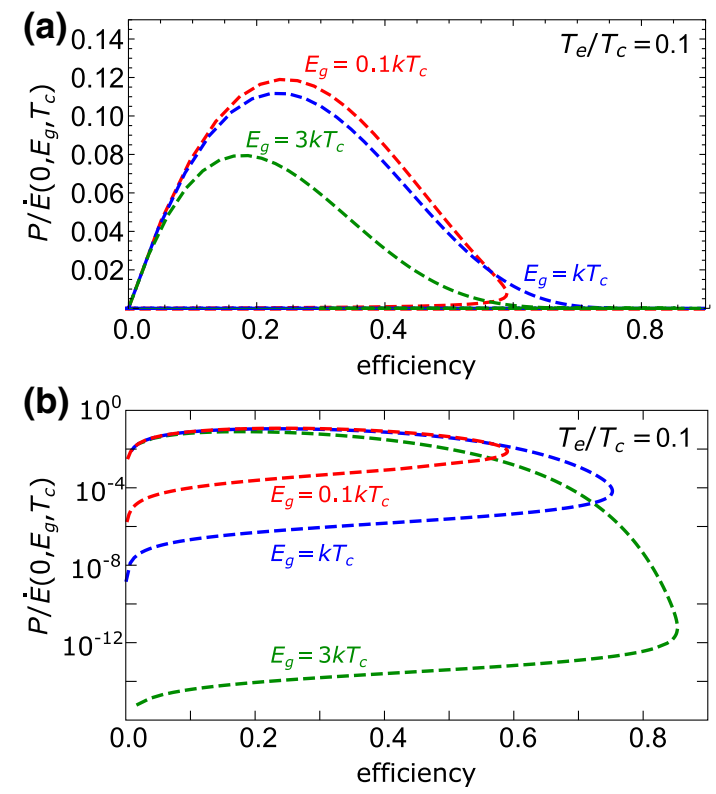

FIG. 4. The TRD power-efficiency characteristic for several values of $E_{g}$ at $T_{e} / T_{c}=0.1$. Power density $P$ is normalized by the emitted-energy density at zero bias. (a) Linear ordinate scale. (b) Logarithmic ordinate scale for better discerning the loop-type (doubled-valued) behavior.

to balance recombination and generation rates is reduced, and Eq. (12) instead becomes

$I(V)=\left[\dot{N}\left(q V, E_{g}, T_{c}\right)-\dot{N}\left(0, E_{g}, T_{e}\right)\right]+\left[R_{\mathrm{nr}}(q V)-G_{\mathrm{nr}}\right]$.

The nonradiative recombination rate $R_{\mathrm{nr}}$ depends on both the bias and device properties. From detailed balance, the nonradiative generation rate $G_{\mathrm{nr}}$ must be equal to the equilibrium nonradiative recombination rate; that is, $G_{\mathrm{nr}}=R_{\mathrm{nr}}(q V=0)$. For the estimates that follow, we ignore any bias dependence of $G_{\mathrm{nr}}$ (which could, for example, stem from impact ionization in a heavily doped semiconductor) and treat the device's external luminescent efficiency $\eta_{\text {ext }}$, defined as

$$
\eta_{\mathrm{ext}}=\frac{\dot{N}\left(q V, E_{g}, T_{c}\right)}{\dot{N}\left(q V, E_{g}, T_{c}\right)+R_{\mathrm{nr}}(q V)}
$$

as constant (corresponding to an ideality factor of unity in a diode equation).

The $I-V$ relation is then

$$
\begin{aligned}
I(V)= & q\left[\frac{\dot{N}\left(q V, E_{g}, T_{c}\right)}{\eta_{\mathrm{ext}}}+\left(1-\eta_{\mathrm{ext}}^{-1}\right) \dot{N}\left(0, E_{g}, T_{c}\right)\right. \\
& \left.-\dot{N}\left(0, E_{g}, T_{e}\right)\right]
\end{aligned}
$$
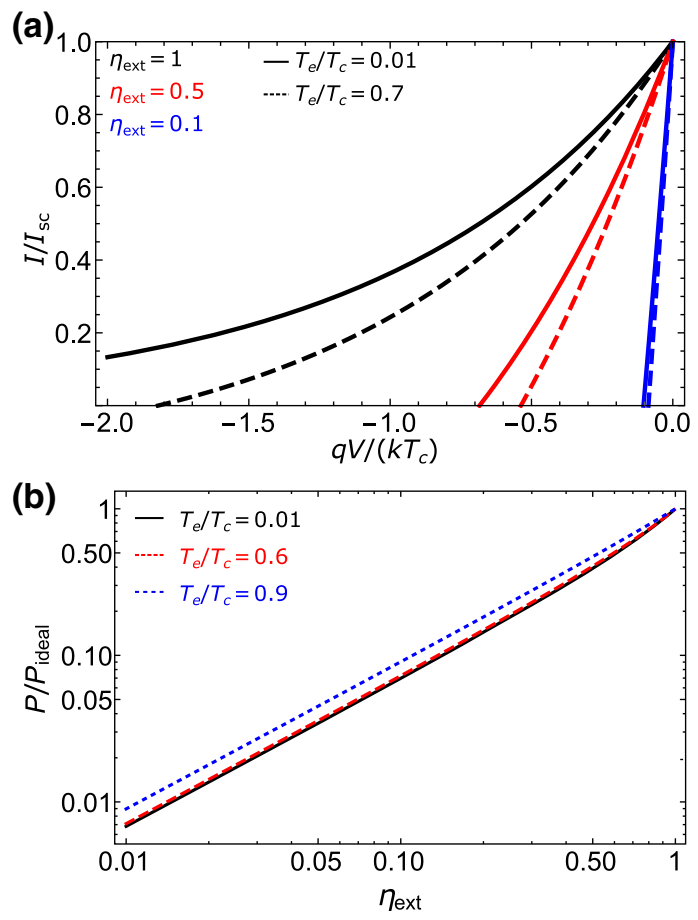

FIG. 5. (a) TRD $I-V$ curves (with current normalized to its short-circuit value) for two values of $T_{e} / T_{c}$ (distinguished by solid vs dashed curves) and three values of external luminescent efficiency. (b) Log-log plot of power for the nonideal TRD normalized to that of the ideal TRD as a function of external luminescent efficiency $\eta_{\text {ext }}$ for three values of $T_{e} / T_{c}$. In both plots, $E_{g}=3 T_{c}$.

Although $I_{\mathrm{sc}}$ is unaffected by $\eta_{\mathrm{ext}}, V_{\mathrm{mpp}}$ is markedly reduced as the external luminescent efficiency decreases [see Fig. 5(a)]. Describing nonradiative generation phenomenologically as a shunt resistance yields a linear $I-V$ relation, which is close to the behavior observed for TRDs realized to date [17] and correctly reproduced by Eq. (22) for small $\eta_{\text {ext }}$. Both $V_{\text {mpp }}$ and power $P$ [see Fig. 5(b)] decrease approximately linearly with $\eta_{\text {ext }}$ as it is reduced. This stands in stark contrast to the logarithmic dependence with which they decrease in a solar cell [18]. TRD performance is thus far more sensitive to material imperfections and requires a rear mirror of high specular reflectivity and light-trapping surfaces or a high-refractive-index extractor that is optically coupled to the diode's emitting surface.

\section{CONCLUSIONS}

In conclusion, we derive fundamental efficiency and power-production bounds for far-field radiative hot-side heat engines that use radiative emission to produce work, first for the far-more-constraining case of black-body (thermal) radiation and then generalized to athermal $(\mu \neq 0)$ radiative emission, which permits higher efficiency. These bounds are elucidated in detail for the TRD, and it is shown why, contrary to understandings predicated exclusively on 
radiative cold-side heat engines (where the presence of radiation transfer introduces unavoidable and irreversible entropy transfer), the Carnot limit can be approached in principle [3] (and hence the nominal Landsberg-efficiency limit can be exceeded) despite the presence of radiative exchange. Akin to basically all known heat engines, the TRD exhibits its own distinct power-efficiency characteristic (trade-off), which is derived and quantified, along with the extreme sensitivity of TRD performance to nonradiative processes and device imperfections. The thermodynamic limits derived here provide bounds for other hot-side engines that may materialize in the future for converting radiative emission into work.

\section{ACKNOWLEDGMENTS}

A.M. acknowledges funding by the Engineering and Physical Science Research Council grant Joint UK-India Clean Energy Centre (Grant No. EP/P003605/1). J.M.G. gratefully acknowledges the hospitality and support of the Institute of Advanced Studies of the University of Western Australia as well as the generous hospitality of N.J.E.-D. at the University of New South Wales during part of the period of this research. N.J.E.-D. acknowledges useful discussions with Martin Green.

[1] P. T. Landsberg and G. Tonge, Thermodynamic energy conversion efficiencies, J. Appl. Phys. 51, R1 (1980).

[2] S. J. Byrnes, R. Blanchard, and F. Capasso, Harvesting renewable energy from earth's mid-infrared emissions, Proc. Natl. Acad. Sci. 111, 3927 (2014).

[3] Rune Strandberg, Theoretical efficiency limits for thermoradiative energy conversion, J. Appl. Phys. 117, 055105 (2015).

[4] Aaswath P. Raman, Wei Li, and Shanhui Fan, Generating light from darkness, Joule 3, 2679 (2019).

[5] S. V. Boriskina, J. K. Tong, W. C. Hsu, B. Liao, Y. Huang, V. Chiloyan, and G. Chen, Heat meets light on the nanoscale, Nanophotonics 5, 134 (2016).

[6] Wei-Chun Hsu, Jonathan K. Tong, Bolin Liao, Yi Huang, Svetlana V. Boriskina, and Gang Chen, Entropic and nearfield improvements of thermoradiative cells, Sci. Rep. 6, 34837 (2016).
[7] Heylal Mashaal and Jeffrey M. Gordon, Basic limit for the efficiency of coherence-limited solar power conversion, Opt. Lett. 39, 5130 (2014).

[8] Tom Markvart, From steam engine to solar cells: Can thermodynamics guide the development of future generations of photovoltaics? Wiley Interdiscip. Rev.: Energy Environ. 5, 543 (2016).

[9] Siddharth Buddhiraju, Parthiban Santhanam, and Shanhui Fan, Thermodynamic limits of energy harvesting from outgoing thermal radiation, Proc. Natl. Acad. Sci. 115, E3609 (2018).

[10] P. Wurfel, The chemical potential of radiation, J. Phys. C: Solid State Phys. 15, 3967 (1982).

[11] Antonio Luque and Antonio Martí, Entropy production in photovoltaic conversion, Phys. Rev. B 55, 6994 (1997).

[12] Ivan Latella, Agustń Pérez-Madrid, J. Miguel Rubi, SvendAge Biehs, and Philippe Ben-Abdallah, Heat Engine Driven by Photon Tunneling in Many-body Systems, Phys. Rev. Appl. 4, 011001 (2015).

[13] Chungwei Lin, Bingnan Wang, Koon Hoo Teo, and Zhuomin Zhang, Near-field enhancement of thermoradiative devices, J. Appl. Phys. 122, 143102 (2017).

[14] William Shockley and Hans J. Queisser, Detailed balance limit of efficiency of p-n junction solar cells, J. Appl. Phys. 32, 510 (1961).

[15] Martin A. Green, Limiting photovoltaic monochromatic light conversion efficiency, Prog. Photovoltaics: Res. Appl. 9, 257 (2001).

[16] J. M. Gordon and Mahmoud Huleihil, General performance characteristics of real heat engines, J. Appl. Phys. 72, 829 (1992).

[17] Masashi Ono, Parthiban Santhanam, Wei Li Bo Zhao, and Shanhui Fan, Experimental demonstration of energy harvesting from the sky using the negative illumination effect of a semiconductor photodiode, Appl. Phys. Lett. 114, 161102 (2019).

[18] O. D. Miller, E. Yablonovitch, and S. R. Kurtz, Strong internal and external luminescence as solar cells approach the shockley-queisser limit, IEEE J. Photovoltaics 2, 303 (2012).

Correction: Equation (9) contained minor typographical errors and has been fixed.

Second Correction: Equation (22) contained a sign error and has been fixed. 